\title{
Correction to: Temporal Stability of Sexual Excitation and Sexual Inhibition in Women
}

\author{
Julia Velten ${ }^{1}\left(\mathbb{D} \cdot\right.$ Lisa Zahler $^{1} \cdot$ Saskia Scholten ${ }^{1} \cdot$ Jürgen Margraf ${ }^{1}$
}

Published online: 1 March 2019

○) Springer Science+Business Media, LLC, part of Springer Nature 2019

\section{Correction to: Archives of Sexual Behavior https://doi.org/10.1007/s10508-018-1323-3}

The authors would like to issue a correction for a statement made in the conclusion section of the paper. As one might expect and as rightly stated throughout the paper, our study suggests that improvements in sexual function might increase sexual excitation and decrease sexual inhibition in women, not the other way around.

Publisher's Note Springer Nature remains neutral with regard to jurisdictional claims in published maps and institutional affiliations.

The original article can be found online at https://doi.org/10.1007/ s10508-018-1323-3.

Julia Velten

julia.velten@rub.de

1 Clinical Psychology and Psychotherapy, Mental Health Research and Treatment Center, Ruhr-Universität Bochum, Massenbergstr. 9-13, 44787 Bochum, Germany 